\title{
Aseguramiento público en salud para población en extrema pobreza y excluida en Honduras ${ }^{1}$
}

\author{
Christian Samuel Canizales Miranda ${ }^{2}$ \\ UNITEC, Tegucigalpa, Honduras.
}

(Recibido: Diciembre, 2014 y Aceptado: Julio 2015)

\begin{abstract}
Resumen:
El objetivo de la investigación es determinar la factibilidad financiera y operativa en la creación de un seguro de salud para personas en extrema pobreza y grupos excluidos en Honduras. Se analizaron experiencias en países de la región y los modelos utilizados para su adaptación en Honduras. La población seleccionada está limitada a ocho departamentos que son: Choluteca, Copán, Gracias a Dios, Intibucá, La Paz, Lempira, Santa Bárbara y Yoro. Se definieron las prestaciones en salud de acuerdo a las necesidades básicas de la población y al marco de salud del país. El modelo de seguro de salud propuesto es un modelo de gestión de propiedad privada con afiliación cerrada, las fuentes de financiación del modelo serán compulsivas y las formas de pago a los prestadores son por financiación a la demanda, las formas de atención por provisión indirecta sin libre elección del prestador y con las prestaciones reguladas por el estado. El pago estimado por persona para el seguro de salud es de L. 1,466.00 para el primer año de la implementación del seguro, el pago para el total de población estimado es de L. 2,714,454,728.00 que representa aproximadamente el 21\% del presupuesto aprobado para la Secretaría de Salud en el 2014. En resumen, el pago por persona está en el rango de los US \$70 que la Secretaría de Salud en promedio gasta actualmente por persona.
\end{abstract}

Palabras Claves: Sistemas, Salud, Prima

\begin{abstract}
:
The study seeks to determine the financial and operational feasibility in the creation of health insurance for people living in extreme poverty and segregated groups in Honduras. Experiences and models from other countries in the region were analyzed for their adaptation to Honduras. The population selected is limited to eight departments or states: Choluteca, Copán, Gracias a Dios, Intibucá, La Paz, Lempira, Santa Bárbara, and Yoro. The research defines health benefits according to the population's basic needs and the country's health framework. The proposed health insurance model is a private held company with closed memberships, compulsive funding sources, payments to the providers based on the demand, services provided by indirect provision with no free choice by the provider, and state regulated benefits. The estimated health insurance fee per person is L. 1,466 for the first year of implementation and the estimated payment for the total population is L. 2,714,454,728, which represents approximately $21 \%$ of the approved budget for the Ministry of Public Health in 2014. In summary, the payment per person is approximately US $\$ 70$, which is the current average spent by the Secretary of Public health.
\end{abstract}

Keywords: Systems, Health, Premium

\section{Introducción}

La salud es la base de toda sociedad. Una población saludable, es una población productiva. Por eso la importancia de los planes de salud accesibles para toda la

\footnotetext{
${ }^{1}$ Articulo ganador del Segundo Lugar en la categoría de Estudiantes de Postgrado del Concurso de Investigación UNITEC-CEUTEC 2014.

${ }^{2}$ Autor para correspondencia. Email: christiancanizales@hotmail.com
} 
población. En Honduras, según cifras de la Encuesta Nacional en Demografía y Salud (ENDESA), el 88\% de las personas no tiene ningún tipo de seguro de salud, porcentaje que se incrementa en el área rural.

Actualmente los servicios de salud gratuitos o subsidiados están a cargo principalmente de la Secretaría de Salud (SESAL) y del Instituto Hondureño de Seguridad Social (IHSS). Pero a lo largo de los años han demostrado ser completamente ineficientes en el manejo de los recursos y los servicios. Es por eso que en el presente estudio se propone una nueva metodología en el manejo de los recursos y prestación de los servicios.

Otro de los problemas que se presenta actualmente con los prestadores de los servicios de salud, es que están focalizados en las principales ciudades del país, dejando prácticamente sin cobertura las zonas rurales y de mayor pobreza. La falta de medicamentos y el bajo personal en los laboratorios se suman a la lista de problemas que enfrenta el actual sistema de salud.

La propuesta de esta investigación, se centra principalmente en las zonas donde los servicios de salud son escasos y la población está bajo la línea de pobreza. Según el Instituto Nacional de Estadísticas (INE), aproximadamente el 64\% de la población está bajo la línea de pobreza, porcentaje que se incrementa en el área rural. Por lo tanto la investigación se centrará en aquellos departamentos de Honduras con mayor nivel de pobreza y menor acceso a los servicios de salud, o los denominados Departamentos Priorizados.

\section{Descripción del problema}

En Honduras la carencia de servicios de salud accesibles para toda la población es un problema que hasta el momento no se ha podido resolver, los hospitales públicos están localizados en las mayores ciudades, dejando sin cobertura diferentes poblaciones sin capacidad de cotizar a servicios privados y que no tienen acceso a los servicios públicos.

"Es por eso que se requiere un cambio en el funcionamiento de todas las dependencias de la Secretaría de Salud (SESAL) y se ha creado un nuevo modelo nacional de salud (MNS)” (Secretaría de Salud, 2014). De acuerdo con este nuevo modelo (Secretaría de Salud, 2013): “La reforma del actual Sistema Nacional de Salud requiere, como condición necesaria, definir qué sistema de salud necesita Honduras para poder insertarse con eficacia en el cambio...” (p.7)

Es evidente que el Sistema Nacional de Salud (SNS), necesita cambios sustanciales en su estructura y funcionamiento, para lograr esto se deben, inicialmente, superar tres grandes desafíos (Secretaría de Salud, 2013): “a. Articular el Sistema Nacional de Salud actualmente fragmentado y no regulado [...] b. Atender con efectividad y calidad a la población, [...] c. Incrementar las posibilidades de impacto en el estado de salud de la población [...]” (p. 3)

El acceso del total de la población a los servicios de salud debe ser una prioridad para todos los gobiernos, ante la ineficiencia demostrada a través de la historia por parte de la SESAL. Para la solución de los problemas de salud en Honduras, es necesario proponer modelos innovadores que provean una solución eficiente y que sean financieramente viables. La SESAL está buscando nuevas formas de afrontar este problema (Secretaría de Salud, 2013): "El diseño del Modelo Nacional de Salud está fundamentado en la visión de una institución descentralizada, con una gestión de recursos centrada en la obtención de resultados, con la capacidad de toma de decisiones apropiadas en forma oportuna, [...]” (p. 5) 
El problema es: cómo incorporar a la población en extrema pobreza, grupos excluidos y grupos autóctonos, en un plan de aseguramiento público subsidiado por el Estado, que brinde los servicios básicos necesarios y que le permita tener acceso a servicios de promoción, prevención, recuperación y rehabilitación de la salud en condiciones de equidad, eficiencia, continuidad, oportunidad y calidad, que garanticen la incorporación de grupos principalmente que viven en áreas rurales, los más pobres y vulnerables así como los pueblos autóctonos y afro-descendientes. Todo lo anterior en un sistema financieramente sostenible.

\subsection{Hipótesis}

Basado en las investigaciones de la SESAL el 17\% de la población hondureña no tiene acceso a ningún servicio de salud, el SNS necesita cambios y nuevas metodologías para un óptimo funcionamiento, se propone la creación de un sistema de servicios de salud descentralizado y con una amplia cobertura para los grupos poblacionales sin acceso a los servicios públicos y privados y que sea factible su elaboración en términos financieros y actuariales. Se calculará un costo promedio por persona para verificar si es factible y viable la propuesta.

\section{Objetivos}

\subsection{Objetivo general}

Elaborar la propuesta de un sistema de servicios en salud, que beneficie a la población en los departamentos con mayor índice en el nivel de pobreza, y con presencia de grupos autóctonos, que sea altamente eficiente y con coberturas mínimas requeridas para prestadores de servicios. Elaborar las proyecciones de los costos mínimos requeridos para asegurar el acceso a servicios de salud a toda la Población objeto de estudio, definida como los grupos de población sin capacidad de cotizar un régimen de aseguramiento público o privado.

\subsection{Objetivos específicos}

1. Definir los grupos de población a cubrir de acuerdo a criterios de: vulnerabilidad y riesgo, exclusión social, cumplimiento al marco político, legal y normativo del país. Definir los grupos poblacionales por atender, la cantidad y ubicación de acuerdo a criterios de: edad, sexo, ingresos y ubicación geográfica.

2. Proponer opciones de aseguramiento público en salud, considerando experiencias exitosas en la región.

3. Sugerir el conjunto de prestaciones básicas y esenciales a brindar a cada grupo de población a considerar, de acuerdo con los lineamientos del Modelo de Atención a la Salud y demás normativas y consensos nacionales.

4. Realizar estimaciones de los costos de aseguramiento con sus proyecciones para un horizonte mínimo de 5 años.

\section{Marco Teórico}

El Plan de Nación, Visión de País, establece en el objetivo uno: “Honduras habrá creado para el 2038, que todos los hondureños, sobre todo los de menores ingresos tengan acceso igualitarios a servicios de calidad en materia de educación, salud...” (Congreso Nacional de Honduras, 2012, p. 70) La meta 1.4 del objetivo 1 alcanzar el 95\% de cobertura en 
salud al 2038, en todos los niveles del sistema, planteando diversas metas intermedias hasta al final de dicha fecha "Para 2038... Honduras habrá sentado las bases de un sistema de salud, capaz de atender las demandas de toda la población y desarrollar esfuerzos sustantivos para profundizar en su sostenibilidad y calidad" (Congreso Nacional de Honduras, 2012, p. 70)

El Marco Conceptual Político y Estratégico de la Reforma del Sector Salud 2009 concibe la Garantía del Aseguramiento, como la acción de garantizar el acceso universal y equitativo de la población a un conjunto de servicios de salud definido como el "básico" complementado con "planes específicos para grupos especiales", incluyendo aquellos que la legislación establezca; la Secretaría de Salud (2009), plantea el establecimiento del Aseguramiento Universal Mixto, que incluirá la definición de un plan obligatorio de salud, la vigilancia del aseguramiento, la regulación del conjunto de servicios, el establecimiento del sistema de enrolamiento individual o colectivo, la definición de la población mínima a asegurar (equilibrio financiero y dilución del riesgo) y la racionalidad de los costos administrativos.

\section{Metodología}

\subsection{Tipo de estudio}

Es una investigación aplicada, bibliográfica y documental que tiene un enfoque cualitativo y cuantitativo, no experimental, explicativo con un enfoque prospectivo.

\subsection{Fuentes de datos, herramientas de recolección utilizadas y procesamiento}

Las fuentes de datos para el análisis son principalmente de informes publicados por los organismos encargados de las diferentes áreas que conforman la investigación, como la SESAL, INE, OIT, OPS, etc. Por lo que la fuente de datos del estudio es documental. Las herramientas de recolección utilizadas se especifican en cada informe por el autor, por lo que se invita a la lectura de las diferentes fuentes bibliográficas para ampliar este tema. El procesamiento de datos es matemático, por medio de fórmulas estadísticas, actuariales y demográficas principalmente. También se procesaron los datos computacionalmente por medio de aplicaciones como Excel y programas estadísticos como MINITAB.

\section{Establecimiento de un régimen de aseguramiento público de salud en Honduras}

\subsection{Definir los grupos de población a cubrir}

En Honduras las estadísticas en relación con la población y distribución de los grupos étnicos es muy escasa y relativamente desactualizada, "Los Censos en Honduras datan desde 1791, realizados por la Iglesia Católica. En la actualidad se han realizado XVI Censos de Población, siendo el último; el del año 2001.” (INE, 2014, En línea).

Para el análisis tomaremos en cuenta la distribución política del país basada en la más reciente información del INE, "La División Política de Honduras, según el Censo de 2001, tiene 18 Departamentos, 298 Municipios, aproximadamente 3,731 Aldeas y 30,591Caseríos.” (INE, 2014, En línea). 


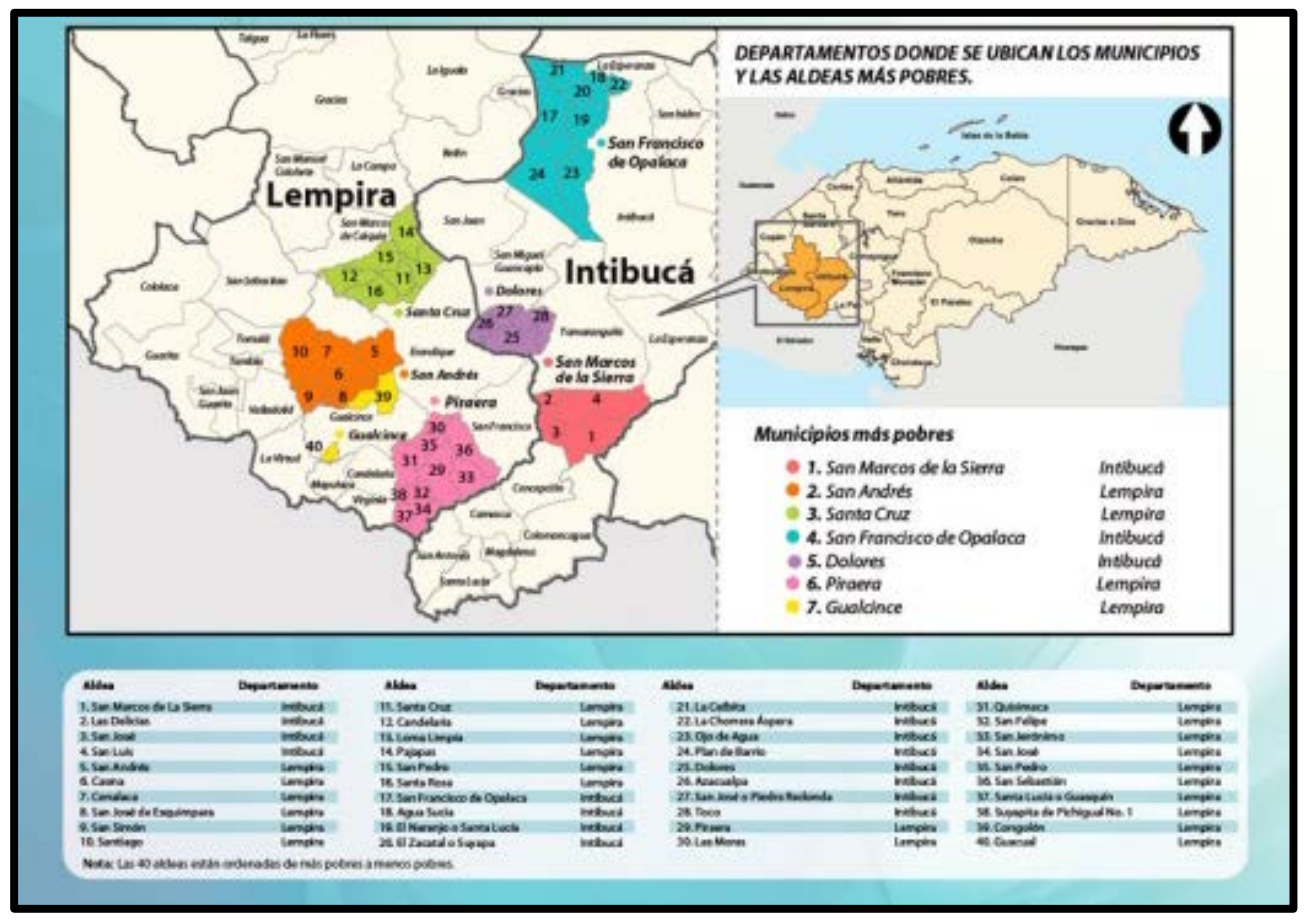

Figura 1. Mapa de los departamentos y municipios de Honduras con las 40 aldeas más pobres del país (INE, 2014).

Para efectos del presente estudio nos centraremos fuera de las urbes y nos enfocaremos principalmente en cuatro tipos de población: población rural excluida, población más pobre y vulnerable, pueblos autóctonos y población afro-descendiente. Según publicaciones del INE (2014), las aldeas más pobres se ubican en los departamentos de Intibucá y Lempira, como podemos observar en la Figura 1.

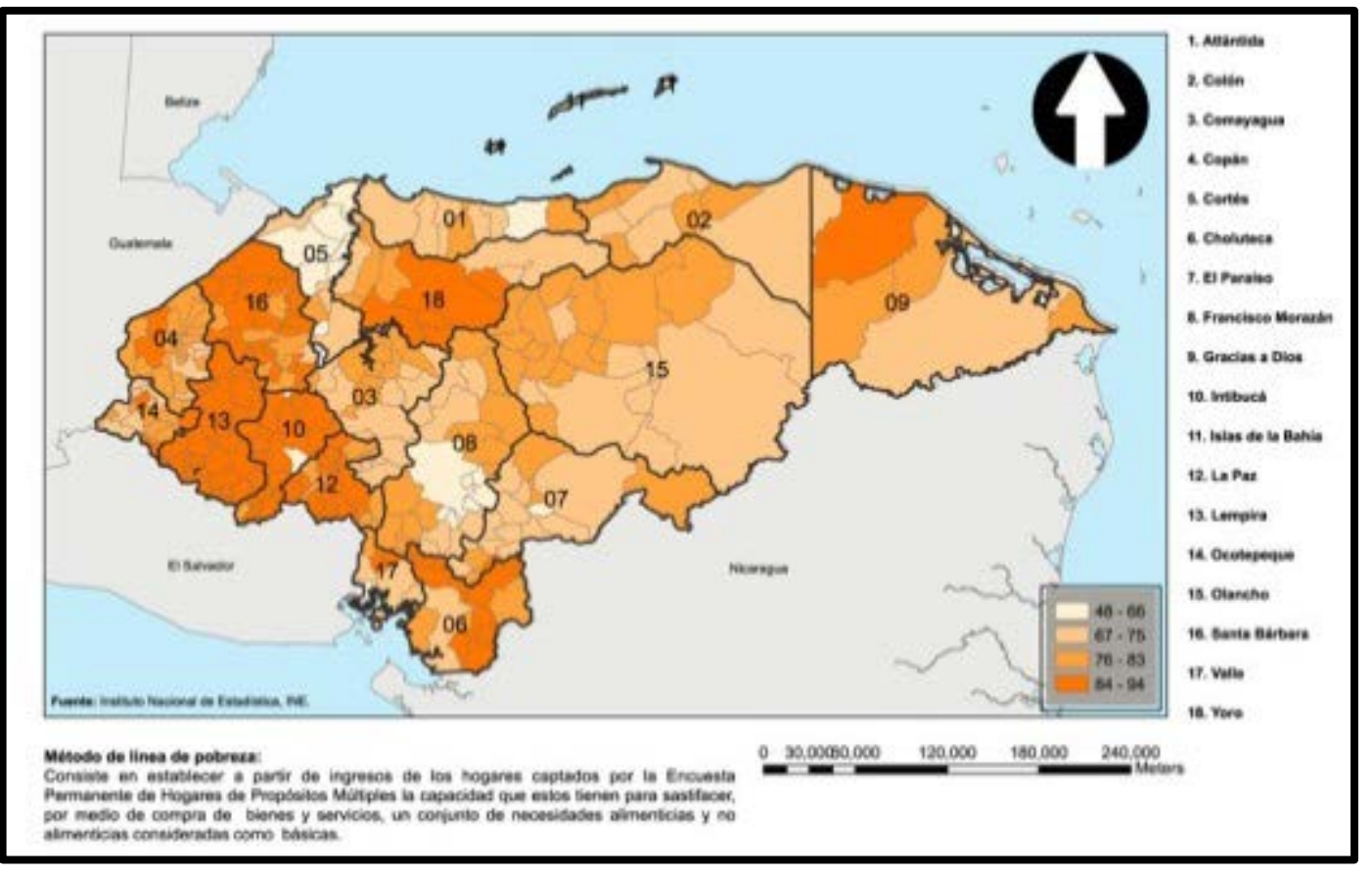

Figura 2. Mapa de Honduras con división municipal clasificado según la línea de pobreza para el año 2002 (INE, 2014). 
Al observar la ubicación geográfica de las aldeas, podemos concluir que es población rural principalmente y sin acceso a los centros de atención pública de las principales urbes, por lo que esta población será parte del estudio. También analizaremos otros departamentos y municipios con poblaciones en extrema pobreza, el mapa de Honduras con su división política municipal clasificada por líneas de pobreza lo observamos en la Figura 2.

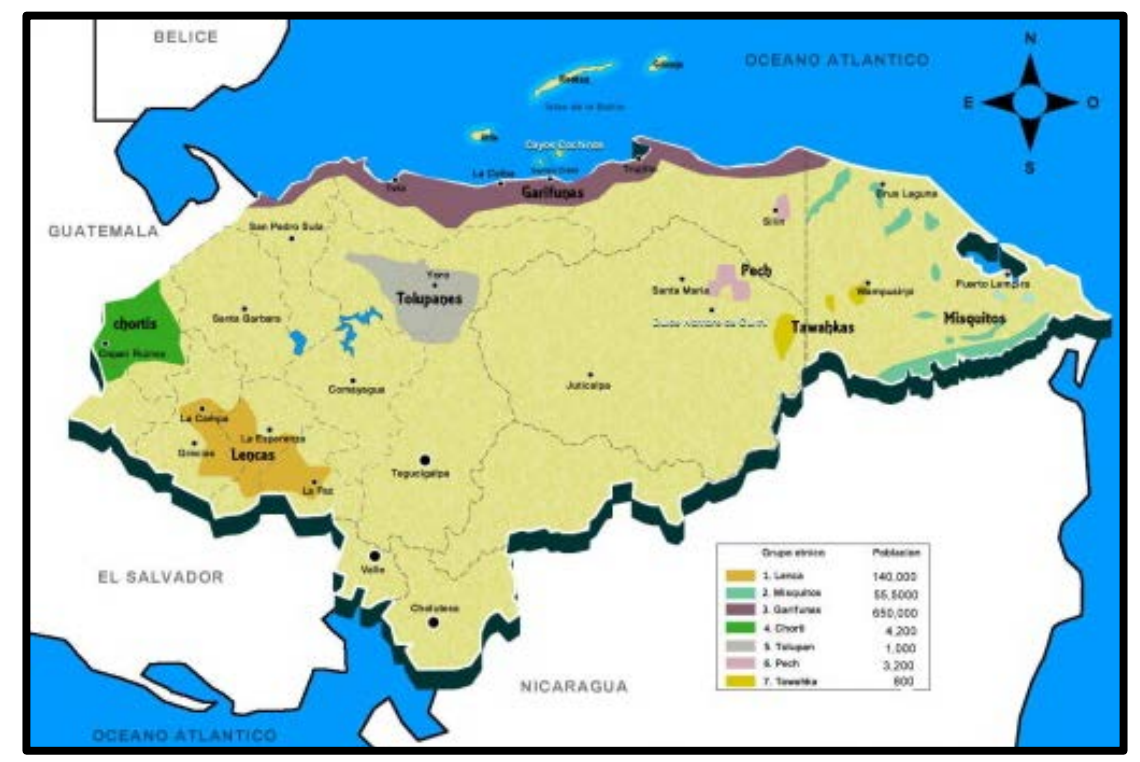

Figura 3. Mapa de Honduras con la división política departamental y concentraciones de grupos étnicos con población aproximada (Ruta Étnica en Honduras, 2014)

Los departamentos con mayor concentración de población bajo la línea de pobreza son: Intibucá, La Paz, Lempira, Santa Bárbara y Yoro seguidos de Choluteca, Gracias a Dios y Copán. En estos departamentos y municipios centraremos la atención del estudio, también vamos a identificar la población autóctona y afro descendiente, la que se encuentra establecida en la Figura 3.

Como podemos observar en la ilustración 3, la mayoría de los asentamientos étnicos de Honduras coinciden en los departamentos de mayor pobreza, con excepción de los Garífunas (Afro-descendientes) que están localizados a lo largo de la costa norte del país, lo que nos facilitará trabajar con cifras de población proyectada para los departamentos en que se encuentran los grupos étnicos y la población bajo la línea de pobreza. Estos grupos étnicos tienen sus particularidades, por ejemplo, algunos hablan su propia lengua, característica a tomar en cuenta al momento de elaborar paquete de servicios de atención.

Basados en lo anterior y con la información del INE por medio de la Secretaria de Salud (2005), aproximadamente 1,194,826 habitantes no tienen acceso a ningún servicio de salud, los cuales ubicaremos en ocho departamentos: Intibucá, La Paz, Lempira, Santa Bárbara, Yoro, Choluteca, Gracias a Dios y Copán, estos pertenecen principalmente a los grupos étnicos: Lencas, Chortís, Garífunas, Tawahka, Tolupanes, Pech y Misquitos, pero que también puede ser población general de esos departamentos que se encuentra en situación de extrema pobreza. 


\subsection{Propuesta de opciones de aseguramiento público en salud}

De acuerdo con la definición de la Organización Mundial de la Salud (OMS), el sistema de salud comprende todas las organizaciones, instituciones y recursos dirigidos a la realización de acción cuyo propósito principal es el mejoramiento de la salud, entre estos se encuentra el Gobierno, profesionales, organizaciones comunitarias y locales, proveedores de servicios entre otros. En Honduras el sistema de salud público está compuesto por la SESAL y el IHSS, que a lo largo de la historia han demostrado ser ineficientes en su funcionamiento, (Secretaría de Salud, 2005): “[...] el actual sistema de salud hondureño se ha fraccionado, desintegrado, débilmente regulado $\mathrm{y}$, como consecuencia, presente alto riesgo de subsidio cruzado, duplicidad de esfuerzos, acciones y gastos. Asimismo, la gestión de los servicios de salud es centralizada y con muy poca coordinación [...]” (p. 26)

Actualmente no se cuenta con un sistema de salud integrado, la SESAL presta servicios a la población no asegurada y en su mayoría de escasos recursos económicos y el IHSS que atiende la población asegurada. Ambas instituciones se centran generalmente en los mismos espacios geográficos y tienen pocos centros de atención. Ambas instituciones con muy baja productividad según estudios de la propia SESAL, (Secretaría de Salud, 2005): "La productividad de la red de atención hospitalaria es baja si se toma en cuenta que acapara el 42\% del presupuesto de la Secretaría de Salud [...]” (p. 26)

El principal problema en el bajo rendimiento se debe a que el sistema de salud en Honduras está centrado en favorecer la oferta y no la demanda de servicios (Secretaría de Salud, 2005): "La baja productividad de los servicios de atención se debe, en parte, a que obedeciendo a lo establecido en la Ley del Estatuto del Médico Empleado, el personal médico labora solamente seis horas diarias [...]” (p. 26)

Aparte de la ineficiencia en los centros de salud operados por el Estado, están también los problemas financieros que enfrentan "Es de importancia notar que, de 2002 a 2005, la Secretaría de Salud se ha visto imposibilitada a mantener abiertos cerca de 130 centros de salud, y que muchos operan bajo condiciones deficientes.” (Secretaría de Salud, 2005) Lo anterior sumado a que estos centros de atención son inaccesibles para las personas en las áreas rurales por cuestiones económicas, según la Secretaría de Salud et al. (2013) en la ENDESA, el 36\% de los hogares del área rural se encuentran en el quintil inferior de la riqueza y solamente un $4 \%$ en el quintil superior, los departamentos que ven incrementado el porcentaje son Lempira con el 60\% de su población en el quintil inferior de la riqueza, Gracias a Dios con el 58\%, Intibucá 53\%.

Según Dinarte (2010) en su estudio de gasto público en salud, señala que el quintil inferior de la riqueza gasta en promedio el $23.58 \%$ de su ingreso en salud mientras el quintil superior únicamente gasta el $4.63 \%$ de su ingreso en salud, lo cual es inequitativo y socialmente injusto, ya que la población con menor ingreso está gastando casi cinco veces más en salud de su ingreso en relación con la clase en el quintil superior de riqueza, lo que contribuye a mantener a esta población en la línea de pobreza, ya que resta de sus ingresos la posibilidad de mejorar sus condiciones de vida. 
Tabla 1: Consumo en salud promedio respecto al ingreso de los hogares por quintil ingreso. Año 2004.

\begin{tabular}{cc}
\hline Quintil de Ingreso del Hogar & $\begin{array}{l}\text { Gasto promedio en salud } \\
\text { / Ingreso en el hogar }\end{array}$ \\
\hline 1 Inferior & $23.58 \%$ \\
2 Segundo & $10.86 \%$ \\
3 Intermedio & $7.88 \%$ \\
4 Cuarto & $6.42 \%$ \\
5 Superior & $4.63 \%$ \\
\hline
\end{tabular}

Fuente: Estudio del gasto público en salud en Honduras. (Dinarte M., 2010, p. 45)

Un dato interesante para análisis es que la población de los departamentos analizados y que presentan mayor población bajo la línea de pobreza, también son los departamentos con mayor porcentaje de población masculina empleada del país: "Intibucá (96 por ciento), El Paraíso (95 por ciento), Lempira (94 por ciento), La Paz, Olancho, Ocotepeque y Santa Bárbara (93 por ciento cada uno), Copán y Comayagua (91 por ciento cada uno).” (Secretaría de Salud et al., 2013, p. 67) Lo que nos hace suponer que el problema de la población no es el desempleo sino el ingreso por tipo de empleo de las familias.

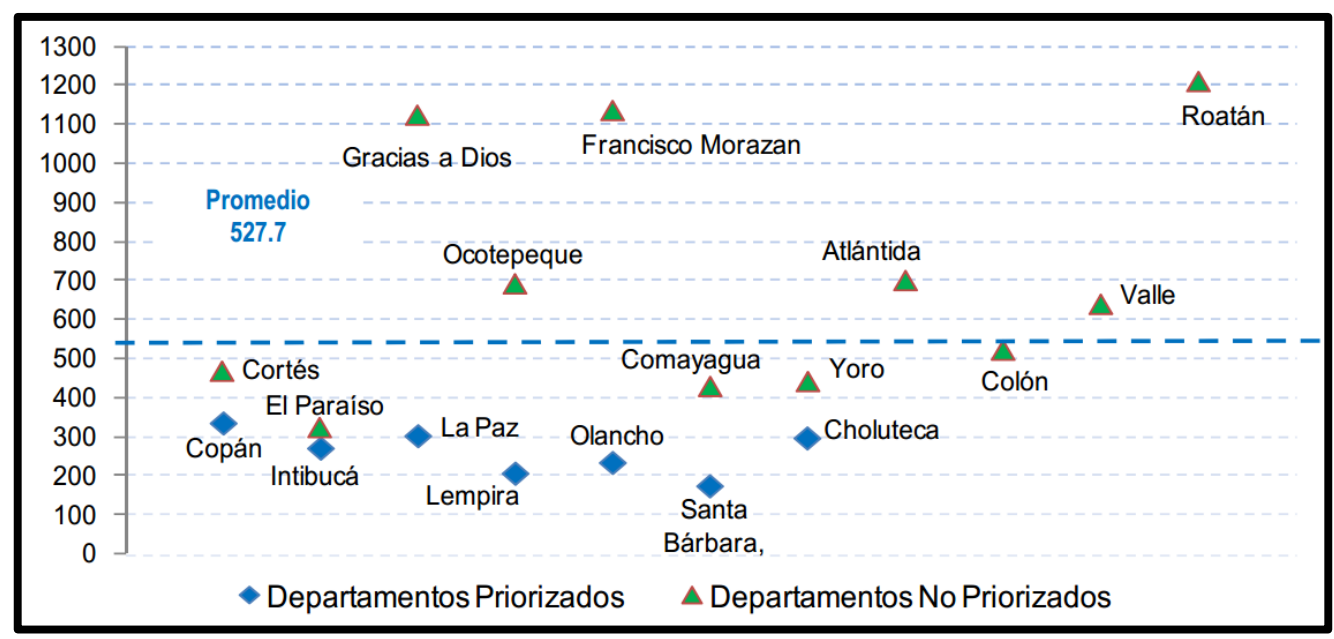

Figura 4. Gasto público per cápita en salud por departamentos, promedio del periodo 2006-2010 en Lempiras. (Dinarte M., 2010, p. 53).

Según la ENDESA, Secretaría de Salud et al. (2013) aproximadamente el 95.4\% de las mujeres que vive en el área rural a nivel nacional no tiene ningún tipo de cobertura de salud, este porcentaje no es muy diferentes en los hombres del área rural de los cuales el 92.8\% no cuenta con ningún tipo de cobertura. Cabe destacar que en el departamento de Lempira el 100\% de los hombres, tanto del área rural como urbana, no cuenta con ningún seguro de salud, seguido por Gracias a Dios con un 99.2\% de la población masculina sin seguro de salud de ningún tipo. Otro aspecto a tomar en cuenta es que el gasto en bienes de capital por parte de la SESAL según Dinarte (2010) en promedio, en el periodo del 2000 al 2010, solamente alcanzó el 3.8\% del total del presupuesto, pero más preocupante aún, es el porcentaje de ejecución de ese presupuesto que solo llegó al 59\% de lo proyectado. Lo anterior se traduce en muy poca inversión en edificios y equipos para el mejoramiento de los servicios prestados o para expandirse a nuevas zonas de atención. 


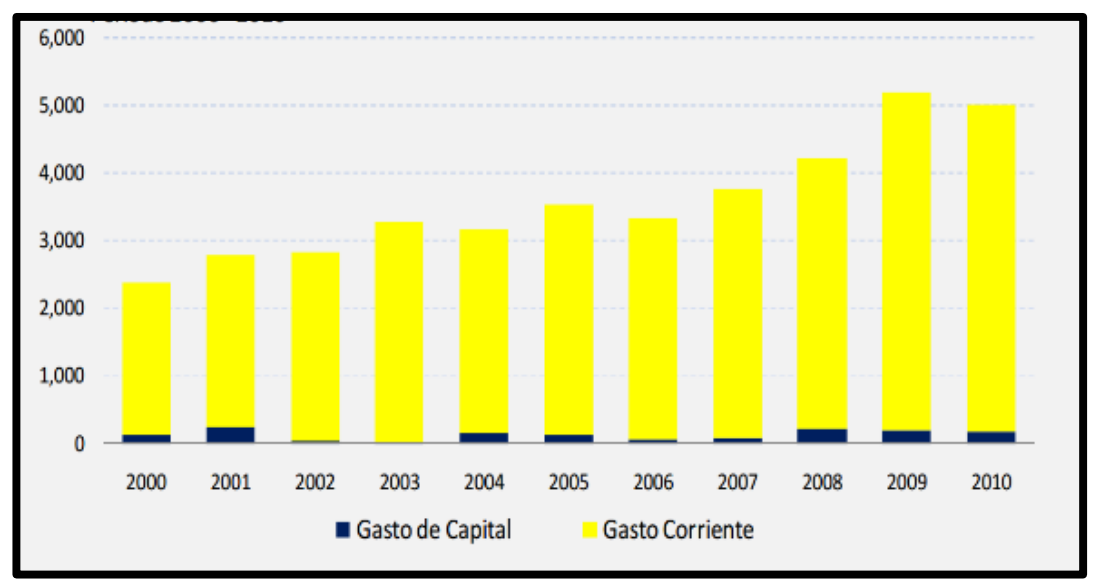

Figura 5. Gasto corriente y de capital de la Secretaría de Salud. (Dinarte, 2010, p. 47).

Otro de los problemas del gasto en salud, además de la poca, por no decir nula, inversión en activos y construcción de nuevos centros; es que gran parte del gasto corriente es en gastos administrativos. Los gastos administrativos componen todos los gastos de la SESAL a nivel central regional y departamental, así como los gastos de función de rectoría y funciones regulatorias entre otras. Según Dinarte (2010), los gastos promedios para el periodo 2000 - 2010 de gestión administrativa fueron el $24 \%$ del total de presupuesto ejecutado, detrás únicamente de los gastos de atención médica hospitalaria con un 39\%, por lo general este gasto siempre es el más alto en los países, pero que la gestión administrativa sea el segundo gasto en importancia sugiere una revisión de los procesos administrativos, ya que otros gastos como la compra de medicamentos solamente es un $10 \%$ del total de presupuesto, muy por debajo en comparación a otros países como Guatemala con el 21.1\%, República Dominicana con 16.6\% y México con $12.3 \%$.

Otro de los problemas graves de la actual estructura de la SESAL, es su distribución del recurso humano; "En el año 2006, la Secretaría de Salud a nivel nacional contaba con un total de 1,037 médicos especialistas, 1,046 médicos generales, enfermeras 1,003, odontólogos 156, auxiliares de enfermería 5,515 y personal administrativo y oficios 2,300 personas.” (Dinarte M., 2010, p. 55) Para el año 2006, el personal administrativo ya superaba el total de médicos especialistas y médicos generales, pero del periodo 2006 2010 según Dinarte (2010), el crecimiento promedio anual del personal administrativo fue del 33.59\% triplicando cualquier otro crecimiento del personal de salud, los médicos generales solamente crecieron en un $13.33 \%$, los médicos especialistas $3.22 \%$, las enfermeras $7.78 \%$, odontólogos $11.45 \%$ y las auxiliares de enfermería con un $3.86 \%$ de crecimiento anual.

Tabla 2. Recurso humano en la Secretaría de Salud.

\begin{tabular}{|c|c|c|c|c|c|c|c|c|c|c|}
\hline Año & $\begin{array}{c}\text { Médico } \\
\text { Especialista }\end{array}$ & $\begin{array}{l}\text { Medico } \\
\text { General }\end{array}$ & Enfermeras & Odontólogos & Psicólogos & $\begin{array}{l}\text { Auxiliar de } \\
\text { Enfermería }\end{array}$ & Técnicos & $\begin{array}{l}\text { Técnicos } \\
\text { Salud } \\
\text { Ambiental }\end{array}$ & $\begin{array}{r}\text { Servicio } \\
\text { de Apoyo }\end{array}$ & $\begin{array}{c}\text { Administrativos } \\
\text { y Oficios }\end{array}$ \\
\hline 2006 & 1,037 & 1,046 & 1,003 & 156 & 46 & 5,515 & 817 & 858 & 3,23 & 2,3 \\
\hline 2007 & 1,1 & 1,15 & 1,068 & 171 & 53 & 5,572 & 891 & 1,383 & 2,664 & 3,09 \\
\hline 2008 & 1,129 & 1,194 & 1,242 & 190 & 54 & 5,975 & 1,001 & 1,299 & 2,781 & 2,834 \\
\hline 2009 & 1,179 & 1,244 & 1,295 & 240 & 54 & 6,323 & 1,075 & 1,299 & 2,848 & 2,838 \\
\hline 2010 & 1,176 & 1,684 & 1,348 & 237 & 52 & 6,408 & 573 & 1,347 & 3,13 & 5,908 \\
\hline
\end{tabular}


El personal administrativo y oficios presentó un crecimiento promedio anual del 34\%, siendo el área que mayor crecimiento experimento, durante el período 2006 al 2010, lo que refleja que se continuará priorizando la contratación de personal en puestos administrativos, en lugar del personal de atención primaria como: médicos, enfermeras y otros, que son los que brindan la atención en salud. Tabla 3 muestra las enfermedades más comunes atendidas por los centros de salud según la ENDESA.

Tabla 3: Enfermedades ambulatorias (últimos 30 días) para las que se buscó asistencia

\begin{tabular}{ccccccccc}
\hline $\begin{array}{c}\text { Caracteristica } \\
\text { (EDAD) }\end{array}$ & $\begin{array}{c}\text { Infecciones } \\
\text { respiratorias }\end{array}$ & $\begin{array}{c}\text { Otras } \\
\text { enfermedades } \\
\text { infecciosas }\end{array}$ & $\begin{array}{c}\text { Enfermedades } \\
\text { crónicas }\end{array}$ & $\begin{array}{c}\text { Parto y } \\
\text { complicaciones } \\
\text { de embarazo }\end{array}$ & $\begin{array}{c}\text { Fracturas/heridas/ } \\
\text { accidentes/violencia }\end{array}$ & $\begin{array}{c}\text { Problemas } \\
\text { dentales }\end{array}$ & $\begin{array}{c}\text { Otras } \\
\text { enfermedades }\end{array}$ & $\begin{array}{c}\text { Sin } \\
\text { información }\end{array}$ \\
\hline$<5$ & 71.70 & 16.80 & 0.00 & 0.00 & 0.60 & 0.10 & 10.50 & 0.20 \\
Total
\end{tabular}

Existen diversos modelos de seguros de salud tales como organizaciones privadas con fines de lucro o seguros sociales financiados con recursos públicos. Hay muchas variaciones de estos tipos de seguros. Tabla 4 muestra una de estas clasificaciones.

Tabla 4: Características distintivas de los seguros de salud sociales y privados

\begin{tabular}{|c|c|c|}
\hline & Sociales & Privados \\
\hline Competencia & Usualmente no compiten & Compiten con precios, cobertura y calidad \\
\hline Plan de beneficios & Único & Diversidad de planes \\
\hline \multirow[t]{2}{*}{ Financiamiento } & - Público (contribuciones e impuestos) & Primas y aranceles \\
\hline & $\begin{array}{l}\text { - Subsidios cruzados a favor de los usuarios } \\
\text { de bajo poder adquisitivo y mala salud }\end{array}$ & \\
\hline \multirow[t]{3}{*}{ Reclutamiento } & - Cerrado & - Voluntario \\
\hline & - Obligatorio-clientela cautiva & - Con libre afiliación \\
\hline & - No seleccionan a sus beneficiarios & $\begin{array}{l}\text { - Pueden practicar selección adversa. Tienden a } \\
\text { concentrarse en aquellos de mayor ingreso y } \\
\text { menor riesgo }\end{array}$ \\
\hline Cobertura & Tiende a ser amplia & Tiende a ser focalizada en determinados grupos \\
\hline Propiedad/lucro & Pública (no tiene fines de lucro) & Privada (tienen fines de lucro) \\
\hline
\end{tabular}

La tecnología administrativa que permitió el funcionamiento de los seguros de salud, fue el desarrollo del cálculo actuarial que produce estimaciones de los valores a ser pagados mensualmente bajo la forma de primas de seguro. Este sistema se denomina prepago. Según Zerda et al. (2001) el país donde el sistema de seguros privados de salud ha alcanzado el mayor desarrollo es Estados Unidos de Norteamérica donde hay más de 1.500 seguros privados.

Según Gonzáles García \& Tobar (1999) en la actualidad cuando se habla de seguros privados o seguros competitivos de salud se hace referencia a un esquema de protección en salud de elección y financiación individual. En este modelo hay ausencia del sector público tanto en las funciones del financiador como de prestador. Tiene una organización típicamente fragmentada, descentralizada y con escasa regulación pública, si bien esta tendencia se está revirtiendo. En comparación con los otros modelos, este modelo de seguros privados limita la acción del Estado a una escasa regulación. 
Según Zerda et al. (2001) se denomina subsidio a la oferta, a la forma más tradicional de financiación en salud. En la América Latina esta modalidad ha sido denominada presupuesto global. El concepto difiere mucho de su acepción europea, ya que en nuestra región se refiere a un modelo muy rígido donde cada establecimiento dispone de un monto fijo por partida, rúbrica o categoría de gasto, que en general no está vinculado con ningún tipo de metas de producción y las autoridades del establecimiento no están habilitadas para reasignar partidas. En contraposición se suele utilizar el término global budget para hacer referencia a un modelo donde se utilizan criterios estrictos para programar el gasto global del establecimiento en función de metas de producción pero la administración del servicio puede reasignar recursos entre partidas.

De acuerdo con Zerda et al. (2001) las ventajas del financiamiento de la oferta radica en que facilita la planificación vertical y otorga a las autoridades sanitarias centrales un máximo control sobre la oferta de recursos facilitando que la asignación de los mismos se relacione con las prioridades sanitarias. Sus desventajas están vinculadas con todas las limitaciones de la centralización administrativa. En el caso específico de Honduras el financiamiento de la oferta es un modelo que ha tenido poca efectividad por diversos factores, entre ellos la corrupción de los administradores de los recursos, también la falta de una estructura administrativa adecuada, ya que usualmente los doctores son quienes dirigen las instituciones y tienen poco conocimiento en temas administrativos y financieros. Las principales alternativas en los componentes de los seguros de salud según Zerda et al. (2001) son las siguientes:

Tabla 5: Componentes de los modelos de los seguros de salud.

\begin{tabular}{|c|c|c|}
\hline Modelos & $\begin{array}{l}\text { Cuestiones } \\
\text { Centrales }\end{array}$ & Opciones \\
\hline \multirow[t]{5}{*}{ Gestión } & Propiedad & - Públicas \\
\hline & & - Privada (lucrativa) \\
\hline & & - No gubernamental ( $\sin$ fines de lucro, cooperativas, sindicales y comunitarias) \\
\hline & Afiliación & - Abierta (universal) \\
\hline & & - Cerrada (focalizada en una determinada categoria de personas) \\
\hline \multirow{6}{*}{ Financiación } & Fuentes & - Voluntarias (primas y mensualidades) \\
\hline & & - Compulsivas (contribuciones, impuestos) \\
\hline & & - Mixtas (combinación de ambas, co-seguros) \\
\hline & Formas de pago & - Financiación de la oferta (presupuesto global, salarios) \\
\hline & a prestadores & $\begin{array}{l}\text { - Financiación de la demanda (a través de formas de pago más o menos } \\
\text { agregadas - desde el pago por prestación hasta las cápitas) }\end{array}$ \\
\hline & & - Contratos de gestión (por resultados) \\
\hline \multirow[t]{6}{*}{ Atención } & Provisión & - Directa (integración vertical) \\
\hline & & - Indirecta (por terceros contratados) con libre elección de prestador \\
\hline & & - Inderecta (por terceros contratados) sin libre elección de prestador \\
\hline & & - Mixtas \\
\hline & Prestaciones & - Definidas libremente desde el mercado \\
\hline & & $\begin{array}{l}\text {-Reguladas por el Estado (plan a minima, parcial o de acuerdo a criterios de } \\
\text { costo-efectividad para la selección). }\end{array}$ \\
\hline
\end{tabular}

En la Tabla 6 se resumen algunos de los países de la región con sus modelos de seguro de salud: 
Tabla 6: Modelos de gestión en los seguros de salud en países seleccionados

\begin{tabular}{|c|c|c|c|c|}
\hline País/Dimensión & $\begin{array}{c}\text { Cobertura } \\
\text { (en millones } \\
\text { habitantes y \%) }\end{array}$ & Cantidad & Propiedad & Reclutamiento \\
\hline \multicolumn{5}{|l|}{ Argentina } \\
\hline OSN & 12 & 293 & $\begin{array}{l}\text { No gubernamental } \\
\text { (generalmente sindical) }\end{array}$ & $\begin{array}{l}\text { Abierto (a partir de la desregulación, hasta } 1993 \text { fue } \\
\text { cerrada por rama de actividad) }\end{array}$ \\
\hline OSP & 4.5 & 23 & Pública & $\begin{array}{l}\text { Cerrado (empleados públicos y sus familiares, aunque } \\
\text { existen afiliados adherentes) }\end{array}$ \\
\hline Prepagas & 2.8 & 281 & Privada & $\begin{array}{l}\text { Abierto - afiliación voluntaria (en general en sectores } \\
\text { de ingresos medios y altos }\end{array}$ \\
\hline \multicolumn{5}{|r|}{$\int_{0}$} \\
\hline R. Contributivo & $7.5(30 \%)$ & 26 & 4 Públicas y 22 Privadas & $\begin{array}{l}\text { Abierto (asalariados con uno o más salarios minimos y } \\
\text { autónomos) }\end{array}$ \\
\hline R. Subsidiado & $8(20 \%)$ & 197 & $\begin{array}{l}2 \text { Públicas, } 15 \text { Privadas y } 180 \\
\text { Comunitarias }\end{array}$ & $\begin{array}{l}\text { Abierto (asalariados con uno o más salarios minimos y } \\
\text { autónomos) }\end{array}$ \\
\hline Medicina prepagada & $2 \%$ & n.d. & Privada & $\begin{array}{l}\text { Abierto - afiliación voluntaria (en general en sectores } \\
\text { de ingresos medios y altos }\end{array}$ \\
\hline \multicolumn{5}{|r|}{$x^{-1}$} \\
\hline Isapres & $26.6 \%$ & n.d. & Privada & $\begin{array}{l}\text { Cerradas ( } 11 \text { y } 1.2 \% \text { de la población) - Abiertas } \\
(25.4 \% \text { de la población) }\end{array}$ \\
\hline Fonasa & $59.5 \%$ & 1 & Pública & Abierto \\
\hline \multicolumn{5}{|l|}{ Costa Rica } \\
\hline CCRSS & $90.4 \%$ & 1 & Pública & Abierto y universal \\
\hline \multicolumn{5}{|l|}{ Guatemala } \\
\hline IGSS & $15.7 \%$ & 1 & Pública & \\
\hline Medicina prepagada & & 12 & Pública & Abierto (libre afiliación) \\
\hline \multicolumn{5}{|l|}{ Estados Unidos } \\
\hline Medicare & $14.2 \%$ & 1 & Pública & Abierto para mayores de 65 años \\
\hline Medicaid & $10.3 \%$ & 1 & Pública & Cerrado para personas de bajos ingresos \\
\hline Seguros privados & $45.3 \%$ & n.d. & Privada & Abierto (libre afiliación) \\
\hline $\mathrm{HMOs}$ & $25.2 \%$ & 651 & Privada & Abierto (libre afiliación) \\
\hline
\end{tabular}

En Guatemala según Zerda et al. (2001), las aseguradoras privadas funcionan desde antes de 1996, aunque fue en este año que se oficializaron. Sin embargo no existe ningún control sobre ellas en cuanto a salud. La Superintendencia de Bancos ejerce vigilancia en relación con sus operaciones financieras (cobro de primas). El criterio de afiliación es abierto universal, cubre alta complejidad a un total de 540.000 beneficiarios, el $23.4 \%$ de todos los atendidos por la seguridad social en salud, con una tendencia creciente. Existe un total de 12 aseguradoras, todas cobran copago, que es variable por tipo de servicios, acordado con afiliados en contrato. No cuentan con instalaciones propias para prestar los servicios, funcionando como intermediarios financieros, reconociendo los gastos en los que los pacientes incurren en centros asistenciales privados. Los servicios son prestados a tarifas fijadas por los hospitales privados, quienes tienen políticas topes para sus médicos, los que pasan facturas de cobro.

Con base en el análisis de los países de la región, la propuesta para Honduras del servicio de salud para la población excluida es la creación de un sistema de prestación de servicios por medio de seguros privados. Para mejorar la eficiencia en la gestión administrativa y garantizar que la mayor cantidad de recursos sea en prestación de servicios de salud y no en gastos administrativos. La SESAL y organismos de financiación externos serán los encargados de aportar los fondos para el pago de la prima de seguro de atención a la población seleccionada. La propuesta en el proceso de pago es que se utilice un fideicomiso por medio del sistema financiero para el manejo de los fondos y evitar los problemas de corrupción en la utilización de recursos, como ocurrió recientemente con el IHSS, el sistema financiero es regulado y auditado por la Comisión Nacional de Bancos y Seguros (CNBS). 


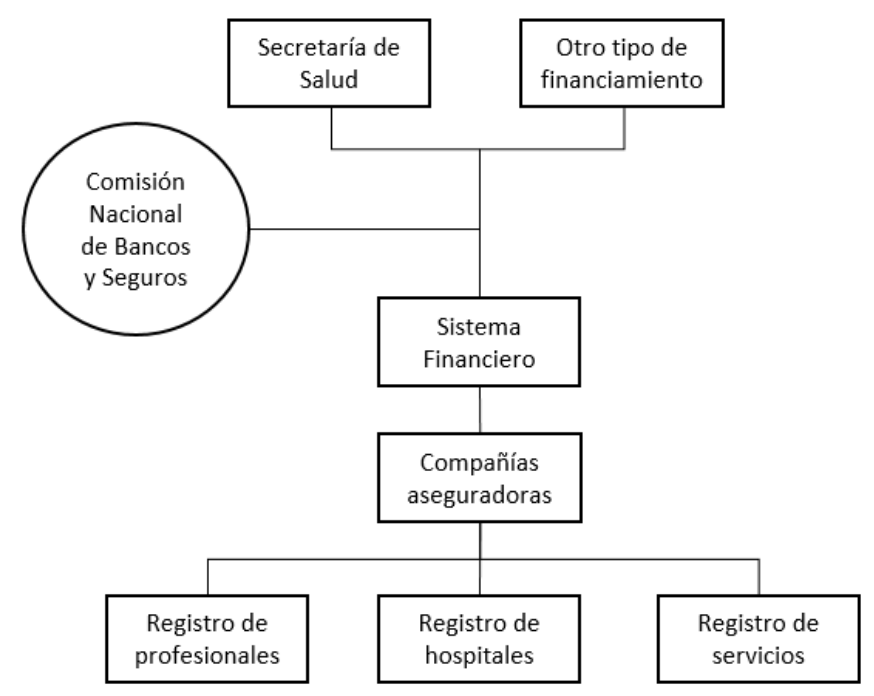

Figura 6. Propuesta de organigrama para los servicios de salud de población excluida.

Se utilizarán aseguradoras privadas, para proveer los servicios de salud a la población final, las cuales deberán estar reguladas debidamente en la CNBS y serán las encargadas de prestar los servicios por su cuenta o tener un registro de personal de salud que cumpla los requisitos mínimos para prestar servicios en las áreas definidas previamente, así como un registro de los hospitales y transporte (como servicios de ambulancias terrestre, marítima, aérea) a los que la población puede tener acceso en caso que sea necesario. En la Figura 6, se presenta el organigrama propuesto.

Se propone un modelo de seguro de salud de gestión privada, por la ineficiencia del sistema público de brindar atención a la población y los altos gastos administrativos en que incurre. El tipo de afiliación será cerrada, ya que solamente se atenderá población rural y excluida de los municipios priorizados que se mencionaron anteriormente, más pobres y vulnerables, pueblos autóctonos y afro-descendientes. Las fuentes de financiación serán de dos tipos ya sea por medio del Estado, a través de la SESAL y otras fuentes de financiamiento, como organismos cooperantes externos, nacionales o privados.

Tabla 7: Propuesta del modelo de seguro de salud para la población excluida hondureña

\begin{tabular}{ccl}
\hline Modelos & $\begin{array}{c}\text { Cuestiones } \\
\text { Centrales }\end{array}$ & \multicolumn{1}{c}{ Opciones } \\
\hline Gestión & $\begin{array}{c}\text { Propiedad } \\
\text { Afiliación }\end{array}$ & - Privada (lucrativa) \\
Financiación & Ferrada (focalizada en una determinada categoria de personas) \\
& Fuentes & - Compulsivas (contribuciones, impuestos) \\
& Formas de pago & - Financiación de la oferta (presupuesto global, salarios) \\
a prestadores & - Financiación de la demanda (a través de formas de pago más o menos \\
& agregadas - desde el pago por prestación hasta las cápitas) \\
Atención & Provisión & $\begin{array}{l}\text { - Inderecta (por terceros contratados) sin libre elección de prestador } \\
\end{array}$ \\
& Prestaciones & -Reguladas por el Estado (plan a minima, parcial o de acuerdo a criterios de \\
& & costo-efectividad para la selección). \\
\hline
\end{tabular}

La forma de pago a los prestadores será por medio de financiación a la demanda, ya que el actual modelo de financiación de la oferta que actualmente maneja la SESAL es ineficiente y se busca un nuevo modelo a atención que mejore los servicios a la población. La provisión de los servicios será de forma indirecta, es decir que se contratarán terceros 
para la prestación de servicios, y se tendrá libre elección del prestador siempre y cuando esté debidamente registrado, esto para fomentar la libre competencia y la mejor atención de la población. El modelo propuesto es de características asistencialistas, con plan de beneficios único, de financiamiento público y con reclutamiento cerrado. En la tabla 6 se presenta un resumen de la propuesta.

\subsection{Costos de las primas y proyecciones financieras}

El pago de las primas de seguros por parte del contratante, en este caso el Gobierno de Honduras pagará para la población beneficiaria, se define según el diccionario MAPFRE de seguros como: "Técnicamente, es el coste de la probabilidad media teórica de que haya siniestro de una determinada clase.” (Fundación MAPFRE, 2014)

La prima debe ser proporcional al riesgo, no equivalente a él, porque depende de un acontecimiento fortuito que puede suceder o no, y cuya cuantía se desconoce a priori. El costo del seguro, o prima comercial, no puede ser solamente el precio teórico de la probabilidad del siniestro, se tienen que considerar otros aspectos como ser: gastos administrativos, gastos de adquisición de la póliza, gastos de redistribución de riesgos y un recargo comercial.

"Lo que conocemos como proceso de riesgo es el proceso estocástico asociado al acontecimiento de los siniestros y a sus respectivas cuantías.” (Hossack et al, 1999). Para expresarlo estadísticamente supongamos que: $\mathrm{N}$ es una variable aleatoria del número de siniestros en el intervalo $t, X_{i}$ es la variable aleatoria cuantía del siniestro i-ésimo para $\mathrm{i}=1, \ldots, \mathrm{N}$. El costo total por indemnizaciones de siniestros acontecidos $\mathrm{S}$ en el periodo $t$, resulta ser: $S=X_{1}+X_{2}+X_{3}+\ldots X_{N}$ cuando $N>0$, y $S=0$ cuando $N=0$. El valor esperado del costo total de la póliza viene dado por: $\mathrm{E}[\mathrm{S}]=\mathrm{E}[\mathrm{N}] \mathrm{E}[\mathrm{X}]$ valor que coincidiría con la prima pura $\mathrm{P}=\mathrm{E}[\mathrm{S}]$.

Esta prima pura $\mathrm{P}$ es la componente fundamental del precio del seguro, ya que está destinada a acumular la recaudación suficiente para hacer frente a los siniestros esperados. Ya hemos establecido que el primer paso dentro del proceso de tarificación a priori es el de selección de las variables de tarifa y sus clases, a partir de unos factores potenciales de riesgo. A continuación vamos a comentar cuáles son las técnicas estadísticas que se emplean usualmente para desempeñar esta labor, terminando así de enmarcar el problema.

Las técnicas del análisis estadístico multivariante son las que permiten organizar procesos de selección teniendo en cuenta simultáneamente el conjunto de factores. El objetivo es obtener un conjunto equilibrado de variables de tarifa, aquel que mejor explique la estructura del riesgo. Al atender una población grande, se considera que las personas que tienen cero o poca siniestralidad compensan las que tienen mayor riesgo, por lo tanto no se considera la estratificación de la población para el cálculo de primas. Las primas de experiencia propia contemplan estos conceptos de la siguiente forma:

$$
\mathrm{PEP}=[\mathrm{S} *(1+\mathrm{I}) *(1+\mathrm{D})] \div[1-\mathrm{GAdq}-\mathrm{GAdm}-\mathrm{U}]
$$

\section{Donde}

PEP = Prima de tarifa de experiencia propia del grupo de negociación.

S = Monto de siniestralidad del grupo de negociación. 


\section{Canizales / Innovare. Vol .4, Núm. 1 (2015) 48 - 69}

I = Factor de ajuste por concepto inflación del sector salud.

$\mathrm{D}=$ Factor de ajuste por concepto de desviaciones en el comportamiento del grupo.

GAdq = Factor de gastos de adquisición asignado al grupo en particular.

GAdm = Factor de gastos de administración estimado para el grupo en particular .

$\mathrm{U}=$ Factor de margen de utilidad esperado para el negocio en particular.

Tabla 8: Honduras: Población censada 1988 y 2001, tasas de crecimiento poblacional según departamento.

\begin{tabular}{lrrr}
\hline \multicolumn{1}{c}{ Departamentos } & \multicolumn{2}{c}{ Población } & $\begin{array}{c}\text { Tasa de Crecimiento } \\
\text { Intercensal (\%) } \\
\text { 1988-2001 }\end{array}$ \\
\hline Copán & $\mathbf{1 9 8 8}$ & $\mathbf{2 0 0 1}$ & 1.8 \\
Choluteca & 227,883 & 288,766 & 1.9 \\
Gracias a Dios & 306,832 & 390,805 & 4.9 \\
Intibucá & 36,313 & 67,384 & 2.6 \\
La Paz & 129,469 & 179,862 & 2.8 \\
Lempira & 109,995 & 156,560 & 2.4 \\
Santa Bárbara & 183,855 & 250,067 & 2.3 \\
Yoro & 289,578 & 342,054 & \\
Total & 346,316 & 465,414 & \\
& & & \\
\hline
\end{tabular}

La población base que se utilizó es la de los dos últimos censos poblacionales según el INE, como población base se utilizó el año 1988 y se calculó la tasa de crecimiento poblacional basado en la tasa de crecimiento geométrico, y como población final se toma la población del censo poblacional del 2001. A continuación se presenta la fórmula utilizada y los resultados:

$$
r=\left(\frac{P^{t+n}}{P^{t}}\right)^{\frac{1}{a}}-1 * 100
$$

Donde

$\mathrm{r}=$ Tasa de crecimiento anual Geométrico.

$\mathrm{P}^{\mathrm{t}}=$ Población al momento inicial.

$\mathrm{P}^{\mathrm{t}+\mathrm{n}}=$ Población al momento $\mathrm{t}+\mathrm{n}$.

$\mathrm{a}=$ La amplitud o distancia en tiempo entre las dos poblaciones. 
Aplicando la tasa de crecimiento intercensal, $P^{t+n}=P^{t}(r / 100)+P^{t}$, se estiman las poblaciones de cada uno de los años intermedios. Una vez calculadas las poblaciones interanuales estimadas con la tasa de crecimiento se proyecta la población para el año 2015, el método elegido para la extrapolación es el de Diferencias Dividas de Newton. Se eligió el método de Newton ya que permite utilizar la información sobre el polinomio de interpolación con $\mathrm{n}$ puntos para calcular el polinomio de interpolación con un argumento más, esto con $\mathrm{n}+1$ puntos. Esto facilita los cálculos al momento de agregar variables al análisis. Para el cálculo se utilizó un polinomio de orden cinco, obtenido con la siguiente fórmula de Newton:

$$
P_{n}(x)=\sum_{i=0}^{n} f\left[x_{0}, x_{1}, \ldots, x_{i}\right] \prod_{j=0}^{i-1}\left(x-x_{j}\right)=\sum_{i=0}^{n} A_{i} \prod_{j=0}^{i-1}\left(x-x_{j}\right)
$$

Que recibe el nombre de fórmula de Newton del polinomio de interpolación. El cálculo de las diferencias divididas se realizará así:

$$
f\left[x_{0}, x_{1}, \ldots, x_{n}\right]=\frac{f\left[x_{1}, x_{2}, \ldots, x_{n}\right]-f\left[x_{0}, x_{1}, \ldots, x_{n-1}\right]}{\left(x_{n}-x_{0}\right)}
$$

Una vez que tenemos las poblaciones esperada para cada año utilizamos la información de la Encuesta Permanente de Hogares de Propósitos Múltiples (EPHPM) 2013, la más reciente publicada hasta el momento, para determinar qué porcentaje de esa población está bajo la línea de pobreza que será la población objetivo del plan de aseguramiento. Según la EPHPM, para el 2013 aproximadamente el 68\% de la población rural a nivel nacional estaba bajo la línea de pobreza y un 60\% de la población urbana. Para obtener la población final objeto de aseguramiento para el 2015 se realiza el siguiente cálculo:

Tabla 9: Honduras: Población urbana y rural bajo la línea de pobreza por departamento.

\begin{tabular}{lcccccccc}
\hline \multirow{2}{*}{ Departamentos } & \multirow{2}{*}{ Población } & \multicolumn{2}{c}{ Población } & \multicolumn{2}{c}{ \% de pobreza } & \multicolumn{2}{c}{ Numero de Personas } & Población \\
& & Rural & Urbana & Rural & Urbana & Rural & Urbana & Objetivo \\
\hline Copán & 372,641 & 174,023 & 198,618 & $68.50 \%$ & $60.04 \%$ & 119,206 & 119,250 & 238,456 \\
Choluteca & 507,109 & 236,820 & 270,289 & $68.50 \%$ & $60.04 \%$ & 162,222 & 162,282 & 324,503 \\
Gracias a Dios & 131,131 & 61,238 & 69,893 & $68.50 \%$ & $60.04 \%$ & 41,948 & 41,964 & 83,912 \\
Intibucá & 256,268 & 119,677 & 136,591 & $68.50 \%$ & $60.04 \%$ & 81,979 & 82,009 & 163,988 \\
La Paz & 228,972 & 106,930 & 122,042 & $68.50 \%$ & $60.04 \%$ & 73,247 & 73,274 & 146,521 \\
Lempira & 348,268 & 162,641 & 185,627 & $68.50 \%$ & $60.04 \%$ & 111,409 & 111,450 & 222,860 \\
Santa Bárbara & 409,250 & 191,120 & 218,130 & $68.50 \%$ & $60.04 \%$ & 130,917 & 130,965 & 261,882 \\
Yoro & 639,854 & 298,812 & 341,042 & $68.50 \%$ & $60.04 \%$ & 204,686 & 204,762 & 409,448 \\
\multicolumn{1}{c}{ Total } & $2,893,493$ & & & & & & & $1,851,570$ \\
& & & & & & & & \\
\hline
\end{tabular}

En términos estadísticos podemos plantear como una probabilidad de sucesos mutuamente excluyentes, ya que si una persona está hospitalizada no se considera una consulta ambulatoria, y si se atiende en una consulta ambulatoria, no está hospitalizada. También pueden considerarse como sucesos independiente ya que la aparición de uno de los sucesos, no está supeditada a la aparición del otro suceso. La probabilidad total estaría representada por: $\mathrm{P}(\mathrm{A}+\mathrm{B})=\mathrm{P}(\mathrm{A})+\mathrm{P}(\mathrm{B})$, donde la probabilidad de presentación conjunta es nula. La probabilidad de A es el número de personas objetivo por la probabilidad de que necesiten una consulta ambulatoria. La probabilidad de B sería el número de personas por la probabilidad que necesiten hospitalización. 
Como ya se estableció el tipo de probabilidad a utilizar se puede calcular el número de expuestos esperados, multiplicando la probabilidad de ocurrencia por el número de personas. Se espera que una persona, en promedio, pueda tener 4 consultas ambulatorias en un año, por lo que se incrementa por 4 el número esperado en esta columna para el cálculo final. En la Tabla 9, podemos ver los resultados.

Tabla 10: Estimación del número total de las reclamaciones esperadas para el año 2015.

\begin{tabular}{|c|c|c|c|c|c|c|}
\hline Departamentos & $\begin{array}{c}\text { Porcentaje de Personas } \\
\text { con Consultas } \\
\text { Ambulatorias }\end{array}$ & $\begin{array}{c}\text { Porcentaje de } \\
\text { Personas que se } \\
\text { Intemo en Hospital }\end{array}$ & $\begin{array}{c}\text { Poblacion } \\
\text { Objetivo por } \\
\text { Departamento }\end{array}$ & $\begin{array}{c}\text { Poblacion Esperada que } \\
\text { Requiere Consultas } \\
\text { Ambulatorias }\end{array}$ & $\begin{array}{c}\text { Poblacion Esperada } \\
\text { que Requiere } \\
\text { Hospitalizacion }\end{array}$ & $\begin{array}{c}\text { Numero de } \\
\text { Reclamaciones } \\
\text { Esperadas en Total }\end{array}$ \\
\hline Copán & $38.6 \%$ & $5.8 \%$ & 238,456 & 92,044 & 13,830 & 382,007 \\
\hline Choluteca & $39.6 \%$ & $5.6 \%$ & 324,503 & 128,503 & 18,172 & 532,186 \\
\hline Gracias a Dios & $34.1 \%$ & $5.5 \%$ & 83,912 & 28,614 & 4,615 & 119,071 \\
\hline Intibucá & $42.5 \%$ & $4.7 \%$ & 163,988 & 69,695 & 7,707 & 286,487 \\
\hline La Paz & $38.2 \%$ & $5.9 \%$ & 146,521 & 55,971 & 8,645 & 232,529 \\
\hline Lempira & $35.5 \%$ & $5.1 \%$ & 222,860 & 79,115 & 11,366 & 327,827 \\
\hline Santa Bárbara & $39.0 \%$ & $5.1 \%$ & 261,882 & 102,134 & 13,356 & 421,892 \\
\hline Yoro & $38.2 \%$ & $5.7 \%$ & 409,448 & 156,409 & 23,339 & 648,975 \\
\hline Total & & & $1,851,570$ & & & $2,950,972$ \\
\hline
\end{tabular}

La probabilidad de ocurrencia del evento así como los costos promedio de cada uno se obtuvieron de información de la ENDESA en los cuadros: 15.2, 15.8 y 15.9.2. (Secretaría de Salud [Honduras], Instituto Nacional de Estadística e ICF International, 2013). Se tomaron los valores de las tablas, para las personas que pagaron por los servicios de salud. $\mathrm{Al}$ igual que en la Tabla 10, se incrementan por 4 los gastos en consultas ambulatorias para calcular el total de gatos, ya que se espera por lo menos este número de ocurrencias durante al año. A continuación se presentan los resultados obtenidos:

Tabla 11: Estimación del gasto total por servicios médicos de la población objetivo.

\begin{tabular}{|c|c|c|c|c|c|c|}
\hline Departamentos & $\begin{array}{c}\text { Porcentaje de Personas } \\
\text { con Consultas } \\
\text { Ambulatorias } \\
\end{array}$ & $\begin{array}{c}\text { Porcentaje de } \\
\text { Personas que se } \\
\text { Interno en Hospital } \\
\end{array}$ & $\begin{array}{c}\text { Poblacion } \\
\text { Objetivo por } \\
\text { Departamento } \\
\end{array}$ & $\begin{array}{c}\text { Gasto Promedio } \\
\text { en Consulta } \\
\text { Ambulatoria (L) } \\
\end{array}$ & $\begin{array}{c}\text { Gasto Promedio } \\
\text { de Hospital por } \\
\text { Evento (L) } \\
\end{array}$ & $\begin{array}{c}\text { Total de Gastos } \\
\text { por Probabilidad } \\
\text { de Ocurrencia }(\mathrm{L})\end{array}$ \\
\hline Copán & $38.6 \%$ & $5.8 \%$ & 238,456 & 534 & 5,325 & $270,253,242$ \\
\hline Choluteca & $39.6 \%$ & $5.6 \%$ & 324,503 & 319 & 4,035 & $237,295,037$ \\
\hline Gracias a Dios & $34.1 \%$ & $5.5 \%$ & 83,912 & 279 & 6,274 & $60,888,554$ \\
\hline Intibucá & $42.5 \%$ & $4.7 \%$ & 163,988 & 282 & 5,058 & $117,600,180$ \\
\hline La Paz & $38.2 \%$ & $5.9 \%$ & 146,521 & 370 & 3,445 & $112,618,089$ \\
\hline Lempira & $35.5 \%$ & $5.1 \%$ & 222,860 & 339 & 3,873 & $151,300,117$ \\
\hline Santa Bárbara & $39.0 \%$ & $5.1 \%$ & 261,882 & 491 & 6,755 & $290,810,977$ \\
\hline Yoro & $38.2 \%$ & $5.7 \%$ & 409,448 & 476 & 4,445 & $401,542,496$ \\
\hline Total & & & $1,851,570$ & & & $1,642,308,691$ \\
\hline
\end{tabular}

Con la información de la Tabla 10 completamos lo requerido para proceder con el cálculo del monto de la siniestralidad del grupo en negociación, planteado en la fórmula 1. Para esto utilizamos la siguiente fórmula:

$$
S=\frac{\text { Número de Reclamaciones }}{\text { Número de Expuestos }} * \frac{\text { Valor Total de las Reclamaciones }}{\text { Número de Reclamaciones }}
$$

En donde el número de reclamaciones lo obtenemos de la Tabla 9, el número de expuestos de la Tabla 8, que es la población objetivo y el valor total de las reclamaciones de la Tabla 10. Con la fórmula anterior estamos evaluando la frecuencia, en la primera parte, por la severidad en la segunda parte. Sustituyendo estos valores en la fórmula 5 obtenemos:

$$
S=\frac{2,950,972}{1,851,570} * \frac{1,642,308,691}{2,950,972}=886.98
$$


El valor de S se define como la Prima Pura del seguro, a esta prima es necesario agregar todos los costos relacionados con la emisión, administración y manejo del seguro. Para eso regresamos a la fórmula 1 , en que sustituimos lo valores para poder determinar la prima total como se explica a continuación.

Según el BCH la inflación interanual para el 2013, fue de un 5.18\% (Banco Central de Honduras, 2014), la cual utilizaremos para la fórmula 1, ya que se estima que los precios de los servicios de salud incrementarán en proporción a la inflación del país. El factor de ajuste por concepto de desviaciones del comportamiento del grupo se estimó en un 10\%. El factor de gastos de adquisición asignado al grupo es relativamente bajo, ya que no se hará por medio de intermediarios o corredores de seguros, sino que se planea una inscripción masiva por medio de registros especializados y con ayuda de las comunidades y alcaldías donde los servicios son requeridos. Por lo anterior estos gastos se estiman en un 5\%. Los gastos administrativos, basados en experiencia del sector de compañías aseguradoras se estiman en un 20\%. Para finalizar, la utilidad esperada se pretende un 5\% para ser competitivos e incentivar a que las compañías aseguradoras aspiren a prestar sus servicios.

Una vez definido lo anterior, podemos calcular la prima total por persona del seguro, sustituyendo los valores en la fórmula 1, se obtiene el siguiente resultado:

$$
\begin{aligned}
& P E P_{2015}=[886.98 *(1+5.18 \%) *(1+10 \%)] \div[1-5 \%-20 \%-5 \%] \\
& =1,466
\end{aligned}
$$

El resultado anterior nos muestra la prima total esperada por persona para el año 2015. Asumiendo que se puede captar el total de la población objetivo para el primer año, el costo total del seguro sería simplemente la población objetivo para el 2015 por la prima total por persona para ese año. A continuación la estimación del pago total esperado para el seguro:

$$
\text { Pago Total } 2015=1,466 * 1,851570=2,714,454,728
$$

El monto anterior es la estimación del pago total de la póliza que se deberá realizar para el año 2015. Para proyectar los siguientes años, se sigue el mismo procedimiento asumiendo que la inflación permanecerá en ese mismo nivel, ya que según la información del $\mathrm{BCH}$, se ha mantenido constante en los últimos años. Utilizando las poblaciones se proyectan los costos de las pólizas de los años siguientes como sigue:

$$
\begin{aligned}
& \text { Pago total }_{2016}=1,537 * 1,892,496=2,909,024,514 \\
& \text { Pago total }_{2017}=1,608 * 1,934,423=3,110,815,757 \\
& \text { Pago total }_{2018}=1,679 * 1,977,379=3,320,073,091 \\
& \text { Pago total }_{2019}=1,750 * 2,021,391=3,537,049,737
\end{aligned}
$$

Para calcular el costo total de la póliza se considera una inflación similar para todos los años siguientes (Para más detalles sobre las proyecciones, ver anexos). El objetivo de la presente investigación es la implementación del seguro de salud para personas excluidas, por lo que el cálculo de la prima es de relevancia para el análisis, las reservas resultan de importancia para la compañía que prestará los servicios de aseguramiento más que para el que los contrata. El presente análisis se realiza desde el punto de vista del contratante por lo que únicamente se mencionarán las reservas requeridas por la CNBS, para un seguro de salud. 
Según la CNBS, en la circular No.003/2004, se establece que las compañías aseguradoras deberán crear las siguientes reservas: Reservas para Riesgo en Curso, Reservas para Siniestros Pendientes de Liquidación, Reservas para Siniestros Ocurridos y no Reportados, Reservas de Previsión y Reservas para Riesgos Catastróficos. (Comisión Nacional de Bancos y Seguros, 2014)

Los costos por persona y totales de la póliza permiten estimar la posibilidad de poder llevar a cabo la propuesta. Según el presupuesto de la república, para el 2014 el presupuesto aprobado para la Secretaría de Salud asciende a L.12,538 millones representando la propuesta, aproximadamente, el 21\% del total del presupuesto en salud.

\section{Conclusiones}

Los grupos de población a cubrir son aquellos que se encuentran bajo la línea de pobreza estimada para cada departamento del país. Se estima que el $60 \%$ de la población urbana vive bajo la línea de pobreza y el 68\% de la población rural. Las poblaciones más pobres coinciden con los departamentos con mayor población de grupos étnicos en el país. Estos departamentos son los priorizados para la atención y son los siguientes: Copán, Choluteca, Gracias a Dios, Intibucá, La Paz, Lempira, Santa Bárbara y Yoro.

Es preciso el cambio en los modelos de servicios de salud, para cambiar de una gestión pública a una privada. En el que, en lugar de financiar la oferta se financiará la demanda, por medio de recursos del Estado o donaciones. Con una afiliación cerrada, es decir, solamente las poblaciones seleccionadas bajo la línea de pobreza que no tengan acceso a ningún otro tipo de aseguramiento y que estén en los departamentos priorizados, podrán formar parte del grupo de asegurados. Las prestaciones de este seguro serán reguladas por el Estado, por medio de acuerdos con las compañías aseguradoras, con una propuesta de servicios mínimos a cubrir. La provisión de estos servicios será indirecta, es decir, por terceros, contratados sin libre elección del prestador, ya que únicamente los registrados para tal fin podrán brindar estos servicios.

La propuesta de prestaciones mínimas se estableció tomando en cuenta las necesidades básicas de cobertura de la población, así como necesidades a futuro que puedan surgir. El grupo de asegurados, al ser un grupo considerable, permite realizar negociaciones en beneficios de los mismos, como ser periodos de espera más cortos o sin periodos de espera para enfermedades preexistentes, servicios de transporte más completos para los asegurados, fácil acceso a las medicinas y clínicas, edades de aceptación más amplias, entre otras.

Respondiendo la hipótesis y con base en experiencias similares de la región, se concluye que si es posible la creación de un sistema de servicios de salud eficiente en cuanto a estructura y costos, que beneficie a población excluida de los servicios tradicionales, públicos y privados, y con costos accesibles. Se estima un costo promedio por asegurado de L.1,500.00 que está dentro del rango del gasto per cápita anual en salud de Honduras, el que se estima en \$US70 (Aproximadamente L.1470.00) anuales. (Dinarte M., 2010, p. 41)

Los beneficios de un plan de aseguramiento de este tipo son muchos, uno de los principales es que los riesgos se transfieren a la compañía aseguradora ya que ella es la responsable de los pagos de los siniestros. Otro beneficio es que, resulta más fácil 
programar los gastos de salud del Estado, basado en primas de seguros que en financiar la oferta. También está demostrado, ampliamente, que en Honduras la Secretaría de Salud y el IHSS, son incapaces de manejar eficientemente los servicios de salud, ventaja que los seguros privados han demostrado a través de la experiencia.

El Estado debe analizar las asignaciones de presupuesto a salud, según Dinarte (2010) Honduras es el país centroamericano con menor gasto anual en salud per cápita de la región. Lo que puede representar un obstáculo para la implementación de la propuesta ya que el presupuesto sería limitado a las aportaciones del Estado y donaciones. La propuesta estima que el costo total de las primas representaría aproximadamente, el $21 \%$ del presupuesto total de la SESAL aprobado para el 2014.

Se debe fortalecer el sistema financiero, específicamente el sector asegurador, por medio de leyes y reglamentos que faciliten su funcionamiento, ya que es el actor principal de la propuesta. El éxito de la propuesta depende de la eficiencia de las compañías aseguradoras y de su capacidad para abarcar sectores nuevos, hasta el momento desatendidos completamente en relación a coberturas de seguros de salud, lo que supone una metodología nueva para este grupo.

Se debe fortalecer la CNBS en recursos humanos y capacidad técnica, encargada de la supervisión de las instituciones financieras. La supervisión tiene una parte fundamental para evitar la corrupción y la malversación de fondos, que ha sido una constante en las gestiones de salud llevadas a cabo por los organismos de gobierno.

\section{Bibliografía}

Anderson, Black, Hair, \& Tatham. (1999). Análisis Multivariante. Prentice Hall.

Arcia, G. (2006). Informe de Focalización. Tegucigalpa: Programa de Red Solidaria.

Banco Central de Honduras. (2013). Programa Monetario 2013-2014. Tegucigalpa: Banco Central de Honduras.

Banco Central de Honduras. (1 de septiembre de 2014). Banco Central de Honduras. Obtenido de Índice de Precios al Consumidor: http://www.bch.hn/download/ipc_historico/ipcm912004.pdf

BID, BIRF, CEPAL, OPS/OMS, OEA, FNUAP, UNICEF, USAID. (1995). Reunión Especial sobre Reformas del Sector Salud. Washington D.C.: División de Salud y Desarrollo Humano.

Boj del Val, E., Claramunt Bielsa, M., \& Fortiana Gregori, J. (2004). Análisis multivariante aplicado a la selección de factores de riesgo en la tarificación. Cuadernos de la Fundación MAPFRE Estudios, Nº 88.

Comisión Nacional de Bancos y Seguros. (1 de septiembre de 2014). CNBS. Obtenido de Comisión Nacional de Bancos y Seguros: http://www.cnbs.gob.hn/files/circulares/2004/C0032004.htm

Congreso Nacional de Honduras. (11 de diciembre de 2012). Plan de Nación 2010 2022. Obtenido de Plan de Nación 2010 - 2022: http://plandenacion.hn/ 
De Vicente, M., Manera Bssa, J., \& Blanco, F. (2000). Análisis Multivariante para las Ciencias Sociales. Madrid: Dykinson.

Dinarte M., M. (2010). Estudio de Gasto Público en Salud en Honduras en el Marco de la Iniciativa de Salud Mesoamérica 2015. Tegucigalpa: Independiente.

Epp, J. (1986). "Lograr la salud para todos". Publicación científica N 557. Organización Panamericana de la Salud.

Fundación MAPFRE. (28 de Agosto de 2014). MAPFRE. Obtenido de DICCIONARIO MAPFRE DE SEGUROS:

http://www.mapfre.es/wdiccionario/general/diccionario-mapfre-seguros.shtml

Gonzáles García, G., \& Tobar, F. (1999). Más salud por el mismo dinero. Buenos Aires: Ediciones Isalud. 2da edición corregida y aumentada.

IHSS. (2004). Boletín Estadístico.

INE. (12 de abril de 2014). Instituto Nacional de Estadística. Obtenido de INE Web site: http://www.ine.gob.hn/drupal/

Instituto Nacional de Estadísticas. (2013). ENCUESTA PERMANENTE DE HOGARES DE PROPÓSITOS MÚLTIPLES. Tegucigalpa: INE.

LÓPEZ-ACUÑA, D., Brito, P., Crocco, P., Alberto, I., José María, M., \& José Luis, Z. (2000). Hacia una nueva generación de reformas para mejorar la salud de las poblaciones. Revista Panamericana de Salud Pública, Vol. 8 Nos. 1 y 2.

Naciones Unidas. (16 de Abril de 2014). Bienvenido a las Naciones Unidas. Obtenido de Declaración Universal de los Derechos Humanos:

http://www.un.org/es/documents/udhr/

Nieto, U., \& Vegas, J. (1993). Matemática actuarial. Editorial MAPFRE S.A.

Ruta Étnica en Honduras. (18 de Abril de 2014). Etnias en Honduras. Obtenido de Rutas Étnicas en Honduras:

http://www.hondurastouristoptions.com/tour_etnico.php

Secretaría de Salud. (10 de Abril de 2014). Secretaría de Salud. Obtenido de http://www.salud.gob.hn/index.html

Secretaría de Salud [Honduras], Instituto Nacional de Estadística e ICF International. (2013). Encuesta Nacional de Salud y Demografía 2011-2012. Tegucigalpa: SS, INE e ICF International.

Secretaria de Salud. (2004). Boletín de Información Estadística de Atención Ambulatoria 2003. Tegucigalpa.

Secretaría de Salud. (2005). Plan Nacional de Salud 2021. Tegucigalpa: Empresa Nacional de Artes Gráficas.

Secretaría de Salud. (2005b). Boletín de Información Estadística de Atención Hospitalaria 2004. Tegucigalpa. 
Secretaría de Salud. (2009). MARCO CONCEPTUAL POLÍTICO Y ESTRATÉGICO DE LA REFORMA DEL SECTOR SALUD 2009. Tegucigalpa: Empresa Nacional de Artes Gráficas.

Secretaría de Salud. (2009). Plan Nacional de Salud 2010-2014. Tegucigalpa: Empresa Nacional de Artes Gráficas.

Secretaría de Salud. (2013). "Por una Honduras saludable" Modelo Nacional de Salud. Tegucigalpa: Secretaría de Salud.

Zerda, Á., Velásquez, G., Tobar, F., \& Vargas, J. (2001). Sistemas de seguros de salud y acceso a medicamentos - Estudios de casos de Argentina, Colombia, Costa Rica, Chile, Estados Unidos de América y Guatemala. Washington D.C.: Pan American Health Organization.

\begin{abstract}
"LA REVISTA INNOVARE NO SE HACE RESPONSABLE EN NINGÚN CASO DE LOS CONTENIDOS, DATOS, CONCLUSIONES U OPINIONES VERTIDAS EN LOS ARTÍCULOS PUBLICADOS, SIENDO ESTA RESPOSABILIDAD EXCLUSIVA DEL (DE LOS) AUTOR (AUTORES)”
\end{abstract}

